\title{
Mutations in PI3K/AKT pathway genes and amplifications of PIK3CA are associated with patterns of recurrence in gastric cancers
}

\author{
Wen-Liang Fang1,2, Kuo-Hung Huang 1,2,3, Yuan-Tzu Lan 2,4, Chien-Hsing Lin ${ }^{5}$, Shih- \\ Ching Chang 2,4,*, Ming-Huang Chen ${ }^{2,6}$, Yee Chao ${ }^{2,6}$, Wen-Chang Lin',8, Su-Shun Lo ${ }^{2,9}$, \\ Anna Fen-Yau $\mathrm{Li}^{2,10}$, Chew-Wun Wu ${ }^{1,2}$, Shih-Hwa Chiou ${ }^{3,11,12}$, Yi-Ming Shyr ${ }^{1,2, *}$ \\ ${ }^{1}$ Division of General Surgery, Department of Surgery, Taipei Veterans General Hospital, Taipei City, Taiwan \\ ${ }^{2}$ School of Medicine, National Yang-Ming University, Taipei City, Taiwan \\ ${ }^{3}$ Institute of Clinical Medicine, School of Medicine, National Yang-Ming University, Taipei City, Taiwan \\ ${ }^{4}$ Division of Colon \& Rectal Surgery, Department of Surgery, Taipei Veterans General Hospital, Taipei City, Taiwan \\ ${ }^{5}$ Genome Research Center, National Yang-Ming University, Taipei City, Taiwan \\ ${ }^{6}$ Division of Hematology and Oncology, Department of Medicine, Taipei Veterans General Hospital, Taipei, Taiwan \\ ${ }^{7}$ Institute of Biomedical Sciences, Academia Sinica, Taipei, Taiwan \\ ${ }^{8}$ Institute of Biotechnology in Medicine, National Yang-Ming University, Taipei, Taiwan \\ ${ }^{9}$ National Yang-Ming University Hospital, Yilan City, Taiwan \\ ${ }^{10}$ Department of Pathology, Taipei Veterans General Hospital, Taipei City, Taiwan \\ ${ }^{11}$ Department of Medical Research and Education, Taipei Veterans General Hospital, Taipei City, Taiwan \\ ${ }^{12}$ Institute of Pharmacology, National Yang-Ming University, Taipei City, Taiwan \\ *These authors have contributed equally to this work \\ Correspondence to: Shih-Ching Chang, e-mail: changsc@vghtpe.gov.tw \\ Yi-Ming Shyr, e-mail: ymshyr@vghtpe.gov.tw \\ Keywords: PI3K/AKT pathway, PIK3CA amplifications, recurrence pattern, diffuse-type, prognosis \\ Received: August 08, $2015 \quad$ Accepted: December 05, $2015 \quad$ Published: December 17, 2015
}

\section{ABSTRACT}

Mutations in genes involved in the PI3K/AKT pathway and amplifications of the PIK3CA gene in gastric cancer and their associations with clinicopathological characteristics and EBV infection were analyzed in this study. A total of 431 patients with gastric adenocarcinomas were enrolled, and 39 mutation hotspots were evaluated in these patients using MALDI-TOF mass spectrometry were analyzed. PIK3CA amplifications were analyzed using real-time quantitative PCR. Regarding patients with intestinal-type gastric cancer, those with mutations in PI3K/AKT pathway genes were also more likely to have tumors located in the lower-third of the stomach than were those without mutations. Regarding patients with diffuse-type gastric cancer, those with PI3K/AKT pathway mutations were more likely to have tumors located in the upper-third of the stomach and to have more hematogenous metastases, particularly in the liver and lungs, than were patients without such mutations (22.2\% vs. $4.5 \%)$. No significant survival difference was observed between patients with vs. without PI3K/AKT pathway mutations. Mutations in PI3K/ AKT pathway genes were associated with hematogenous metastasis in patients with diffuse-type gastric cancer. Only when the tumors were located in the middle-third of stomach, tumor with mutations of the PIK3CA gene or mutations of the PI3K/ AKT pathway genes were associated with more EBV infection than those without mutations. Patients with PIK3CA amplifications were more likely to have diffusetype and poorly differentiated gastric cancers and were more likely to experience peritoneal recurrence compared with those without PIK3CA amplifications. Even 


\section{upon subgroup analysis, PI3KCA amplifications were found to not affect the patients' outcomes.}

\section{INTRODUCTION}

Although the incidence of gastric cancer has continued to decline worldwide, it remains the fourth most common cancer and the second most common cause of cancer death worldwide [1]. Approximately $90 \%$ recurrence occurs within 3 years after curative surgery for gastric cancer and is generally associated with a poor outcome [2].

It is known that the accumulation of genetic alterations leads to the development of gastric carcinoma [3]. Most of the genetic mutations in gastric carcinoma correlate with changes in biological signals, such as those in the phosphatidylinositol 3-kinase/AKT/mammalian target of the rapamycin pathway (PI3K/AKT/mTOR pathway) [4]. $P I 3 K$ signaling is a crucial regulator of many essential cellular processes, including cell growth, metabolism, survival, metastasis, and resistance to chemotherapy [5]. Although little substantial evidence exists regarding whether the $\mathrm{PI} 3 \mathrm{~K} / \mathrm{AKT}$ pathway is frequently altered in gastric carcinomas, its precise function remains to be determined [5-7]. An understanding of the biological pathways leading to the development of gastric carcinoma will provide an opportunity to improve targeted therapies.

It was reported that $P I 3 K$ and $A K T$ proteins are significantly overexpressed in tumor tissues and in tumors with lymph node metastasis [8]. Furthermore, the simultaneous expression of PI3K, $p$-AKT, and $p-m T O R$ was an independent prognostic factor of a poor outcome [9]. Therefore, investigation of aspects of the PI3K/ AKT/mTOR pathway in gastric cancers may yield useful biomarkers and lead to the development of targeted therapeutic agents for these cancers [10].

PIK3CA mutations and amplifications are two major causes of the overactivation of the PI3K/AKT pathway in gastric cancers [11]. Deregulation of the PI3K/ AKT pathway can occur subsequent to the existence of oncogenic mutations in the PIK3CA gene [12]. The $P I K 3 C A$ gene, which encodes the catalytic subunit pf $P I 3 K$, is mutated at a high frequency in gastric cancer cell lines and tumor tissues [13]. Several studies have demonstrated that somatic mutations in PI3KCA are present in $4 \%-25 \%$ of patients with gastric carcinoma [14-18]. However, there was no association between PIK3CA mutations and clinicopathological features. The correlation between PIK3CA mutations and EBV infection remains controversial; some authors [19] reported a strong correlation, but other authors [20] reported no significant correlation between them. As previously reported [19], EBV-infected gastric cancer was associated with $P I K 3 C A$ mutation, particularly those occurring in the body of the stomach.
Earlier studies reported a higher frequency of PIK3CA amplifications than mutations [11,21-23]. The frequency of $P I 3 K C A$ amplifications has been reported to be $13 \%-67 \%$ [11,21-23]. Some studies [11,21] demonstrated that $P I K 3 C A$ amplifications were associated with a poor prognosis for gastric cancer patients; however, others reported no association with their clinicopathological features [22,23]. Therefore, whether $P I K 3 C A$ amplifications can serve as a prognostic indicator for gastric cancers remains unclear.

In this study, we examined the effects of the mutation spectra of the PI3K/AKT pathway genes in a Chinese population. However, whole-genome sequencing is a time-consuming and expensive analytical method. Therefore, a high-throughput assay is a crucial tool for clinical practices. Based on the previous results of a wholegenome sequencing investigation and the development of MALDI-TOF mass spectrometry for nucleic-acid analysis, we designed a 39 hot-spot Sequenom MassARRAY platform. We used this platform to analyze mutation spectra and molecular features of the PI3K/AKT pathway and used real-time quantitative PCR (qPCR) to investigate $P I K 3 C A$ amplifications in patients with gastric cancer and the associations with clinicopathological characteristics, recurrence patterns and prognoses.

\section{RESULTS}

\section{Clinicopathological characteristics}

\section{Mutations in PI3K/AKT pathway genes}

$\mathrm{PI} 3 \mathrm{~K} / \mathrm{AKT}$ pathway genetic mutations were found in $69(16 \%)$ of the 431 patients. As shown in Figure 1 (A), the most commonly mutated genes were PIK3CA $(13.2 \%)$, followed by PTEN (4.0\%), AKT3 (1.62\%), AKT2 (0.46\%), and $A K T 1(0.23 \%)$.

As shown in Supplementary Table 1, the most prevalent hotspot mutations in the $P I K 3 C A$ gene were c. $1633 \mathrm{G}>A(\mathrm{n}=14)$, followed by c. $3140 \mathrm{~A}>\mathrm{G} \quad(\mathrm{n}=11)$ and c. $1624 \mathrm{G}>\mathrm{A}(\mathrm{n}=10)$, and then by the other mutations shown in the table. The most prevalent hotspot mutations in the PTEN gene were c. $1003 \mathrm{C}>\mathrm{T}(\mathrm{n}=5)$ and c.800delA $(n=5)$, followed by c.518G $>A(n=3)$, and then by the other mutations shown in the table. The most prevalent hotspot mutations in the $A K T 3$ gene were c. $49 \mathrm{G}>\mathrm{A}(\mathrm{n}=4)$, followed by c.496C $>\mathrm{T}(\mathrm{n}=2)$. There were two patients with hotspot mutations in $A K T 2$; one of whom had the c. $1063 \mathrm{C}>\mathrm{T}$ mutation and the other of whom had the c. $904 \mathrm{~A}>\mathrm{G}$ mutation. There was only one patient with the c. $49 \mathrm{G}>\mathrm{A}$ hotspot mutation in the AKT1 gene. All of the hotspot mutations selected for this study were non-silent mutations resulting in an amino acid change, a stop codon or frame shift. 
There were 11 patients with two hotspot mutations, whereas no patient with more than two hotspot mutations. The general data of the 11 patients were shown in Supplementary Table 2. Among the 11 patients, four patients have two hotspot mutations in the same gene, including two patients with two hotspot mutations in $P T E N$ gene and two patients with two hotspot mutations in $P I K 3 C A$ gene. The other seven patients have two hotspot mutations in different genes, including four patients with $P I K 3 C A$ and PTEN mutations, two patients with PTEN and AKT3 mutations, and one patient with PIK3CA and AKT3 mutations. Among the 11 patients, seven patients had tumor recurrence and one of them received another surgery for regional lymph node recurrence and was alive more than 20 years after the second operation. The other six patients with tumor recurrence all died of cancer. Four out of the 11 patients had no tumor recurrence and one of them died of second primary cancer as hepatoma. Three patients were alive without tumor recurrence until the last follow-up.

Among the 11 patients, two patients had simultaneous EBV infection and PIK3CA mutations and both of them had stage III gastric cancer and had tumor recurrence within one year after surgery. Both of them died of tumor recurrence.

The correlation between the clinicopathological characteristics and the mutation status of the PI3K/AKT pathway genes is shown in Table 1 . Of the 69 patients with at least one mutation in the PI3K/AKT pathway genes, 51 had intestinal-type gastric cancer (73.9\%), which was significantly more frequent than in those without any mutations in the PI3K/AKT pathway genes $(184 / 362,50.8 \%, P=0.001)$. No significant difference was observed between these two groups with respect to other clinicopathological characteristics.

Because the biological behaviors of diffuse-type and intestinal-type gastric cancers differ, we separated

(A)
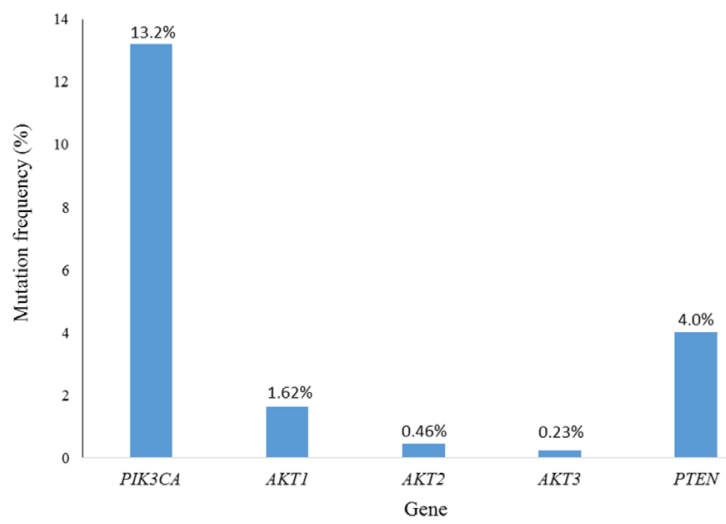

the intestinal-type and diffuse-type gastric cancer cases when analyzing the differences between patients with and without mutations in PI3K/AKT pathway genes.

Among the 431 patients in this study, 235 of them $(54.5 \%)$ had an intestinal-type gastric cancer and 196 of them $(45.5 \%)$ had a diffuse-type gastric cancer. Regarding intestinal-type gastric cancer, patients with PI3K/AKT pathway mutations were more likely to have tumors in the lower-third of the stomach (Table 2). Regarding diffusetype gastric cancer, patients with mutations in the PI3K/ AKT pathway were more likely to have tumors in the upper-third of the stomach (Table 3).

As shown in Table 4, tumors with an EBV infection were more likely to be located in the middlethird of the stomach $(42.2 \%)$, whereas tumors without an EBV infection were more frequently located in the lower-third of the stomach (50.4\%). Moreover, only when the tumors were located in the middle-third of the stomach were tumors with mutations in the PIK3CA gene or in the PI3K/AKT pathway genes more likely to harbor EBV infection than were those without such mutations (Table 5).

\section{PIK3CA amplifications}

Of the 431 patients in this study, PIK3CA amplifications were found in 206 of the patients $(47.8 \%)$. The mean copy number in these patients was $4.33 \pm 1.66$, with a range of 0.3-6.02. As previously reported [11], amplification of the PIK3CA gene was defined as a copy number of $\geq 3$ with a $P$-value of $<0.05$. The correlation between the clinicopathological characteristics and the mutation status of the PI3K/AKT pathway genes is shown in Table 1. A greater percentage of the patients with PIK3CA amplifications had diffuse-type gastric cancer (52.9\% vs. $38.7 \%, P=0.004)$ and poorly differentiated

(B)

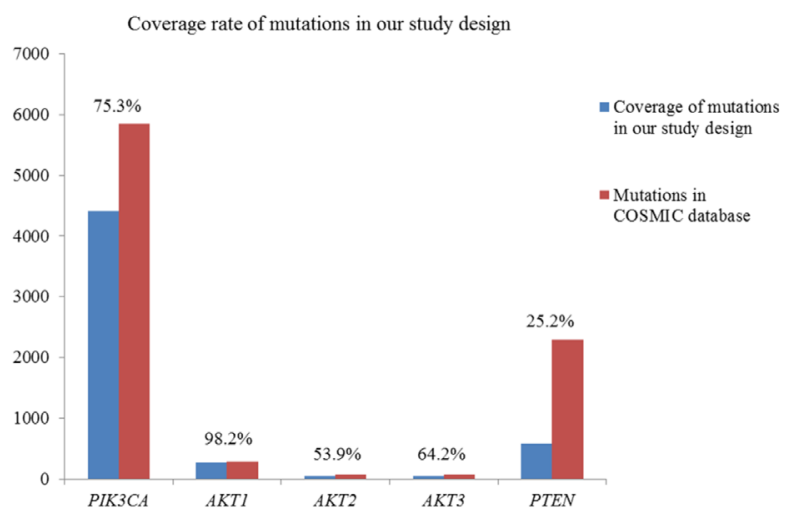

Figure 1: A. The frequency of the selected mutation hot spots in five genes within the PI3K/AKT pathway according to MassARRAY. B. The coverage rate of the selected mutation hot spots in five genes within the PI3K/AKT pathway according to MassARRAY compared with the COSMIC database. 
Table 1: Clinical profile in all gastric cancer patients

\begin{tabular}{|c|c|c|c|c|c|c|}
\hline Variables & $\begin{array}{c}\text { PI3K/AKT pathway } \\
\text { mutation } \\
(-) \\
\text { n=362 } \\
\text { n }(\%)\end{array}$ & $\begin{array}{c}\text { PI3K/AKT } \\
\text { pathway } \\
\text { mutation } \\
(+) \\
\text { n=69 n }(\%)\end{array}$ & $P$ value & $\begin{array}{c}\text { PIK3CA } \\
\text { amplification } \\
(-) \\
\mathrm{n}=225 \\
\mathrm{n}(\%)\end{array}$ & $\begin{array}{c}\text { PIK3CA } \\
\text { amplification } \\
(+) \\
\mathrm{n}=\mathbf{2 0 6} \\
\mathrm{n}(\%)\end{array}$ & $\begin{array}{c}P \\
\text { value }\end{array}$ \\
\hline Age & & & 1.000 & & & 0.493 \\
\hline$<65$ yrs & $147(40.6)$ & $28(40.6)$ & & $95(42.2)$ & $80(38.8)$ & \\
\hline$\geq 65 \mathrm{yrs}$ & $215(59.4)$ & $41(59.4)$ & & $130(57.8)$ & $126(61.2)$ & \\
\hline Gender & & & 0.389 & & & 0.057 \\
\hline Male & $252(69.6)$ & $52(75.4)$ & & $168(74.7)$ & $136(66)$ & \\
\hline Female & $110(30.4)$ & $17(24.6)$ & & $57(25.3)$ & $70(34)$ & \\
\hline Tumor size & & & 0.140 & & & 0.552 \\
\hline$<5 \mathrm{~cm}$ & $145(40.1)$ & $21(30.4)$ & & $90(40)$ & $76(36.9)$ & \\
\hline$\geq 5 \mathrm{~cm}$ & $217(59.9)$ & $48(69.6)$ & & $135(60)$ & $130(63.1)$ & \\
\hline Tumor location & & & 0.750 & & & 0.774 \\
\hline Upper stomach & $69(19.1)$ & $11(15.9)$ & & $45(20)$ & $35(17)$ & \\
\hline Middle stomach & $118(32.6)$ & $23(33.3)$ & & $70(31.1)$ & $71(34.5)$ & \\
\hline Lower stomach & $163(45.0)$ & $34(49.3)$ & & $104(46.2)$ & $93(45.1)$ & \\
\hline Whole stomach & $12(3.3)$ & $1(1.4)$ & & $6(2.7)$ & $7(3.4)$ & \\
\hline Cell differentiation & & & 0.122 & & & 0.031 \\
\hline Poor & $204(56.4)$ & $30(43.5)$ & & $109(48.4)$ & $125(60.7)$ & \\
\hline Moderate & $151(41.7)$ & $38(55.1)$ & & $113(50.2)$ & $76(36.9)$ & \\
\hline Well & $7(1.9)$ & $1(1.4)$ & & $3(1.3)$ & $5(2.4)$ & \\
\hline Gross appearance & & & 0.157 & & & 0.477 \\
\hline Superficial type & $49(13.5)$ & $7(10.1)$ & & $30(13.3)$ & $26(12.6)$ & \\
\hline Borrmann type $1 \& 2$ & $106(29.3)$ & $14(20.3)$ & & $57(25.3)$ & $63(30.6)$ & \\
\hline Borrmann type $3 \& 4$ & $207(57.2)$ & $48(69.6)$ & & $138(61.3)$ & $117(56.8)$ & \\
\hline Stromal reaction type & & & 0.673 & & & 0.138 \\
\hline Medullary type & $56(15.5)$ & $9(13.0)$ & & $40(17.8)$ & $25(12.1)$ & \\
\hline Intermediate type & $189(52.2)$ & $40(58.0)$ & & $121(53.8)$ & $108(52.4)$ & \\
\hline Scirrhous type & $117(32.3)$ & $20(29.0)$ & & $64(28.4)$ & $73(35.4)$ & \\
\hline Lauren's histology & & & 0.001 & & & 0.004 \\
\hline Intestinal type & $184(50.8)$ & $51(73.9)$ & & $138(61.3)$ & $97(47.1)$ & \\
\hline Diffuse type & $178(49.2)$ & $18(26.1)$ & & $87(38.7)$ & $109(52.9)$ & \\
\hline $\begin{array}{l}\text { Lymphovascular } \\
\text { invasion }\end{array}$ & & & 0.885 & & & 0.915 \\
\hline Absent & $102(28.2)$ & $20(29.0)$ & & $63(28)$ & $59(28.6)$ & \\
\hline Present & $260(71.8)$ & $49(71.0)$ & & $162(72)$ & $147(71.4)$ & \\
\hline Pathological T category & & & 0.322 & & & 0.550 \\
\hline $\mathrm{T} 1$ & $61(16.9)$ & $6(8.7)$ & & $32(14.2)$ & $35(17)$ & \\
\hline $\mathrm{T} 2$ & $58(16.0)$ & $11(15.9)$ & & $41(18.2)$ & $28(13.6)$ & \\
\hline T3 & $124(34.3)$ & $24(34.8)$ & & $75(33.3)$ & $73(35.4)$ & \\
\hline $\mathrm{T} 4$ & $119(32.7)$ & $28(40.6)$ & & $77(34.2)$ & $70(34)$ & \\
\hline
\end{tabular}

(Continued) 


\begin{tabular}{|c|c|c|c|c|c|c|}
\hline Variables & $\begin{array}{c}\text { PI3K/AKT pathway } \\
\text { mutation } \\
(-) \\
\text { n=362 } \\
\text { n (\%) } \\
\end{array}$ & $\begin{array}{c}\text { PI3K/AKT } \\
\text { pathway } \\
\text { mutation } \\
(+) \\
\mathrm{n}=69 \mathrm{n}(\%) \\
\end{array}$ & $P$ value & $\begin{array}{c}P I K 3 C A \\
\text { amplification } \\
(-) \\
\text { n=225 } \\
\text { n (\%) } \\
\end{array}$ & $\begin{array}{c}\text { PIK3CA } \\
\text { amplification } \\
(+) \\
\text { n=206 } \\
\text { n (\%) } \\
\end{array}$ & $\begin{array}{c}P \\
\text { value }\end{array}$ \\
\hline $\begin{array}{l}\text { Pathological N } \\
\text { category }\end{array}$ & & & 0.843 & & & 0.490 \\
\hline N0 & $115(31.8)$ & $24(34.8)$ & & $71(31.6)$ & $68(33)$ & \\
\hline $\mathrm{N} 1$ & $58(16.0)$ & $13(18.8)$ & & $42(18.7)$ & $29(14.1)$ & \\
\hline $\mathrm{N} 2$ & $94(26.0)$ & $16(23.2)$ & & $59(26.2)$ & $51(24.8)$ & \\
\hline N3 & $95(26.2)$ & $16(23.2)$ & & $53(23.6)$ & $58(28.2)$ & \\
\hline $\begin{array}{l}\text { Pathological TNM } \\
\text { Stage }\end{array}$ & & & 0.762 & & & 0.591 \\
\hline I & $77(21.3)$ & $12(17.4)$ & & $47(20.9)$ & $42(20.4)$ & \\
\hline II & $102(28.2)$ & $20(29.0)$ & & $68(30.2)$ & $54(26.2)$ & \\
\hline III & $183(50.6)$ & $37(53.6)$ & & $110(48.9)$ & $110(53.4)$ & \\
\hline
\end{tabular}

Table 2: Clinical profile in intestinal-type gastric cancer patients

\begin{tabular}{|c|c|c|c|c|c|c|}
\hline Variables & $\begin{array}{c}\text { PI3K/AKT } \\
\text { pathway mutation } \\
(-) \\
\mathbf{n}=184 \\
\mathbf{n}(\%)\end{array}$ & $\begin{array}{c}\text { PI3K/AKT } \\
\text { pathway } \\
\text { mutation }(+) \\
\mathbf{n}=51 \\
\mathbf{n}(\%)\end{array}$ & $P$ value & $\begin{array}{c}P I K 3 C A \\
\text { amplification } \\
(-) \\
n=138 \\
\text { n }(\%)\end{array}$ & $\begin{array}{c}\text { PIK3CA } \\
\text { amplification } \\
(+) \\
\mathbf{n}=97 \\
\mathrm{n}(\%)\end{array}$ & $P$ value \\
\hline Age & & & 0.736 & & & 0.673 \\
\hline$<65 \mathrm{yrs}$ & $59(32.1)$ & $18(35.3)$ & & $47(34.1)$ & $30(30.9)$ & \\
\hline$\geq 65 \mathrm{yrs}$ & $125(67.9)$ & $33(64.7)$ & & $91(65.9)$ & $67(69.1)$ & \\
\hline Gender & & & 1.000 & & & 0.746 \\
\hline Male & $145(78.8)$ & $40(78.4)$ & & $110(79.7)$ & $75(77.3)$ & \\
\hline Female & $39(21.2)$ & $11(21.6)$ & & $28(20.3)$ & $22(22.7)$ & \\
\hline Tumor size & & & 0.200 & & & 0.503 \\
\hline$<5 \mathrm{~cm}$ & $82(44.6)$ & $17(33.3)$ & & $61(44.2)$ & $38(39.2)$ & \\
\hline$\geq 5 \mathrm{~cm}$ & $102(55.4)$ & $34(66.7)$ & & $77(55.8)$ & $59(60.8)$ & \\
\hline Tumor location & & & 0.028 & & & 0.302 \\
\hline Upper stomach & $42(22.8)$ & $4(7.8)$ & & $24(17.4)$ & $22(22.7)$ & \\
\hline Middle stomach & $51(27.7)$ & $16(31.4)$ & & $39(28.3)$ & $28(28.9)$ & \\
\hline Lower stomach & $90(48.9)$ & $30(58.8)$ & & $74(53.6)$ & $46(47.4)$ & \\
\hline Whole stomach & $1(0.5)$ & $1(2.0)$ & & $1(0.7)$ & $1(1)$ & \\
\hline Cell differentiation & & & 0.451 & & & 0.744 \\
\hline Poor & $33(17.9)$ & $13(25.5)$ & & $26(18.8)$ & $20(20.6)$ & \\
\hline Moderate & $145(78.8)$ & $37(72.5)$ & & $110(79.7)$ & $72(74.2)$ & \\
\hline Well & $6(3.3)$ & $1(2.0)$ & & $2(1.4)$ & $5(5.2)$ & \\
\hline
\end{tabular}

(Continued) 


\begin{tabular}{|c|c|c|c|c|c|c|}
\hline Variables & $\begin{array}{c}\text { PI3K/AKT } \\
\text { pathway mutation } \\
(-) \\
\mathbf{n}=184 \\
\mathbf{n}(\%)\end{array}$ & $\begin{array}{c}\text { PI3K/AKT } \\
\text { pathway } \\
\text { mutation }(+) \\
\mathbf{n}=\mathbf{5 1} \\
\mathbf{n}(\%)\end{array}$ & $P$ value & $\begin{array}{c}P I K 3 C A \\
\text { amplification } \\
(-) \\
\text { n=138 } \\
\text { n }(\%)\end{array}$ & $\begin{array}{c}P I K 3 C A \\
\text { amplification } \\
(+) \\
\mathbf{n}=97 \\
\mathrm{n}(\%)\end{array}$ & $P$ value \\
\hline Gross appearance & & & 0.115 & & & 0.035 \\
\hline Superficial type & $24(13.0)$ & $6(11.8)$ & & $22(15.9)$ & $8(8.2)$ & \\
\hline $\begin{array}{l}\text { Borrmann type } \\
1 \& 2\end{array}$ & $66(35.9)$ & $11(21.6)$ & & $37(26.8)$ & $40(41.2)$ & \\
\hline $\begin{array}{l}\text { Borrmann type } \\
3 \& 4\end{array}$ & $94(51.1)$ & $34(66.7)$ & & $79(57.2)$ & $49(50.5)$ & \\
\hline Stromal reaction type & & & 0.249 & & & 0.171 \\
\hline Medullary type & $21(11.4)$ & $7(13.7)$ & & $21(15.2)$ & $7(7.2)$ & \\
\hline Intermediate type & $133(72.3)$ & $31(60.8)$ & & $92(66.7)$ & $72(74.2)$ & \\
\hline Scirrhous type & $30(16.3)$ & $13(25.5)$ & & $25(18.1)$ & $18(18.6)$ & \\
\hline $\begin{array}{l}\text { Lymphovascular } \\
\text { invasion }\end{array}$ & & & 0.596 & & & 0.883 \\
\hline Absent & $49(26.6)$ & $16(31.4)$ & & $39(28.3)$ & $26(26.8)$ & \\
\hline Present & $135(73.4)$ & $35(68.6)$ & & $99(71.7)$ & $71(73.2)$ & \\
\hline $\begin{array}{l}\text { Pathological T } \\
\text { category }\end{array}$ & & & 0.368 & & & 0.693 \\
\hline $\mathrm{T} 1$ & $33(17.9)$ & $5(9.8)$ & & $20(14.5)$ & $18(18.6)$ & \\
\hline $\mathrm{T} 2$ & $39(12.2)$ & $9(17.6)$ & & $30(21.7)$ & $18(18.6)$ & \\
\hline $\mathrm{T} 3$ & $58(31.5)$ & $17(33.3)$ & & $42(30.4)$ & $33(34)$ & \\
\hline $\mathrm{T} 4$ & $54(29.3)$ & $20(39.2)$ & & $46(33.3)$ & $28(28.9)$ & \\
\hline $\begin{array}{l}\text { Pathological N } \\
\text { category }\end{array}$ & & & 0.861 & & & 0.717 \\
\hline N0 & $71(38.6)$ & $21(41.2)$ & & $56(40.6)$ & $36(37.1)$ & \\
\hline $\mathrm{N} 1$ & $29(15.8)$ & $8(15.7)$ & & $22(15.9)$ & $15(15.5)$ & \\
\hline $\mathrm{N} 2$ & $50(27.2)$ & $11(21.6)$ & & $37(26.8)$ & $24(24.7)$ & \\
\hline N3 & $34(18.5)$ & $11(21.6)$ & & $23(16.7)$ & $22(22.7)$ & \\
\hline $\begin{array}{l}\text { Pathological TNM } \\
\text { Stage }\end{array}$ & & & 0.659 & & & 0.877 \\
\hline I & $49(26.6)$ & $11(21.6)$ & & $35(25.4)$ & $25(25.8)$ & \\
\hline II & $57(31.0)$ & $15(29.4)$ & & 44 (31.9) & $28(28.9)$ & \\
\hline III & 78 (42.4) & $25(49.0)$ & & $59(42.8)$ & $44(45.4)$ & \\
\hline
\end{tabular}

adenocarcinoma $(60.7 \%$ vs. $48.4 \%, P=0.031)$ compared with those patients without PIK3CA amplifications. Regarding intestinal-type gastric cancer, patients with mutations in the PI3K/AKT pathway genes were more likely to have a less superficial-type of gastric cancer (Table 2). Regarding diffuse-type gastric cancer, there was no significant difference between the clinicopathological characteristics of patients with or without mutations in the PI3K/AKT pathway genes (Table 3).

As shown in Table 5, there is no correlation between EBV infection and patients with or without PI3KCA amplifications. 
Table 3: Clinical profile in diffuse-type gastric cancer patients

\begin{tabular}{|c|c|c|c|c|c|c|}
\hline Variables & $\begin{array}{c}\text { PI3K/AKT } \\
\text { pathway } \\
\text { mutation } \\
(-) \\
\text { n=178 } \\
\text { n }(\%)\end{array}$ & $\begin{array}{c}\text { PI3K/AKT } \\
\text { pathway } \\
\text { mutation } \\
(+) \\
\text { n=18 } \\
\text { n }(\%)\end{array}$ & $P$ value & $\begin{array}{c}\text { PIK3CA } \\
\text { amplification } \\
(-) \\
\text { n=87 } \\
\text { n (\%) }\end{array}$ & $\begin{array}{c}P I K 3 C A \\
\text { amplification } \\
(+) \\
\text { n=109 } \\
\text { n }(\%)\end{array}$ & $P$ value \\
\hline Age & & & 0.805 & & & 0.250 \\
\hline$<65$ yrs & $88(49.4)$ & $10(55.6)$ & & $48(55.2)$ & $50(45.9)$ & \\
\hline$\geq 65 \mathrm{yrs}$ & $90(50.6)$ & $8(44.4)$ & & $39(44.8)$ & $59(54.1)$ & \\
\hline Gender & & & 0.801 & & & 0.143 \\
\hline Male & $107(60.1)$ & $12(66.7)$ & & $58(66.7)$ & $61(56)$ & \\
\hline Female & $71(39.9)$ & $6(33.3)$ & & $29(33.3)$ & $48(44)$ & \\
\hline Tumor size & & & 0.308 & & & 0.880 \\
\hline$<5 \mathrm{~cm}$ & $63(35.4)$ & $4(22.2)$ & & $29(33.3)$ & $38(34.9)$ & \\
\hline$\geq 5 \mathrm{~cm}$ & $115(64.6)$ & $14(77.8)$ & & $58(66.7)$ & $71(65.1)$ & \\
\hline Tumor location & & & 0.007 & & & 0.087 \\
\hline Upper third stomach & $27(15.2)$ & 7 (38.9) & & $21(24.1)$ & $13(11.9)$ & \\
\hline Middle third stomach & $67(37.6)$ & $7(38.9)$ & & $31(35.6)$ & $43(39.4)$ & \\
\hline Lower third stomach & $73(41.0)$ & $4(22.2)$ & & $30(34.5)$ & $47(43.1)$ & \\
\hline Whole stomach & $11(6.2)$ & 0 & & $5(5.7)$ & $6(5.5)$ & \\
\hline Cell differentiation & & & 0.850 & & & 0.535 \\
\hline Poor & $171(96.1)$ & $17(94.4)$ & & $83(95.4)$ & $105(96.3)$ & \\
\hline Moderate & $6(3.4)$ & $1(5.6)$ & & $3(3.4)$ & $4(3.7)$ & \\
\hline Well & $1(0.6)$ & 0 & & $1(1.1)$ & 0 & \\
\hline Gross appearance & & & 0.437 & & & 0.324 \\
\hline Superficial type & $25(14.0)$ & $1(5.6)$ & & $8(9.2)$ & $18(16.5)$ & \\
\hline Borrmann type $1 \& 2$ & $40(22.5)$ & $3(16.7)$ & & $20(23)$ & $23(21.1)$ & \\
\hline Borrmann type $3 \& 4$ & $113(63.5)$ & $14(77.8)$ & & $59(67.8)$ & $68(62.4)$ & \\
\hline Stromal reaction type & & & 0.263 & & & 0.592 \\
\hline Medullary type & $35(19.7)$ & $2(11.1)$ & & $19(21.8)$ & $18(16.5)$ & \\
\hline Intermediate type & $56(31.5)$ & $9(50.0)$ & & $29(33.3)$ & $36(33)$ & \\
\hline Scirrhous type & $87(48.9)$ & $7(38.9)$ & & $39(44.8)$ & $55(50.5)$ & \\
\hline Lymphovascular invasion & & & 0.596 & & & 0.752 \\
\hline Absent & $53(29.8)$ & $4(22.2)$ & & $24(27.6)$ & $33(30.3)$ & \\
\hline Present & $125(70.2)$ & $14(77.8)$ & & $63(72.4)$ & $76(69.7)$ & \\
\hline Pathological T category & & & 0.697 & & & 0.855 \\
\hline $\mathrm{T} 1$ & $28(15.7)$ & $1(5.6)$ & & $12(13.8)$ & $17(15.6)$ & \\
\hline $\mathrm{T} 2$ & $19(10.7)$ & $2(11.1)$ & & $11(12.6)$ & $10(9.2)$ & \\
\hline T3 & $66(37.1)$ & $7(38.9)$ & & $33(37.9)$ & $40(36.7)$ & \\
\hline $\mathrm{T} 4$ & $65(36.5)$ & $8(44.4)$ & & $31(35.6)$ & $42(38.5)$ & \\
\hline
\end{tabular}

(Continued) 


\begin{tabular}{|c|c|c|c|c|c|c|}
\hline Variables & $\begin{array}{c}\text { PI3K/AKT } \\
\text { pathway } \\
\text { mutation } \\
(-) \\
\text { n=178 } \\
\text { n }(\%)\end{array}$ & $\begin{array}{c}\text { PI3K/AKT } \\
\text { pathway } \\
\text { mutation } \\
(+) \\
n=18 \\
n(\%)\end{array}$ & $P$ value & $\begin{array}{c}\text { PIK3CA } \\
\text { amplification } \\
(-) \\
\mathrm{n}=\mathbf{8 7} \\
\mathrm{n}(\%)\end{array}$ & $\begin{array}{c}\text { PIK3CA } \\
\text { amplification } \\
(+) \\
\mathrm{n}=109 \\
\mathrm{n}(\%)\end{array}$ & $P$ value \\
\hline Pathological N category & & & 0.583 & & & 0.118 \\
\hline No & $44(24.7)$ & $3(16.7)$ & & $15(17.2)$ & $32(19.4)$ & \\
\hline N1 & $29(16.3)$ & $5(27.8)$ & & $20(23)$ & $14(12.8)$ & \\
\hline N2 & $44(24.7)$ & $5(27.8)$ & & $22(25.3)$ & $27(24.8)$ & \\
\hline N3 & $61(34.3)$ & $5(27.8)$ & & $30(34.5)$ & $36(33)$ & \\
\hline Pathological TNM Stage & & & 0.510 & & & 0.818 \\
\hline I & $28(15.7)$ & $12(5.6)$ & & $12(13.8)$ & $17(15.6)$ & \\
\hline II & $45(25.3)$ & $20(27.8)$ & & $24(27.6)$ & $26(23.9)$ & \\
\hline III & $105(59.0)$ & $37(66.7)$ & & $51(58.6)$ & $66(60.6)$ & \\
\hline
\end{tabular}

Table 4: Clinical profile in gastric cancer patients with/without EBV infection

\begin{tabular}{|c|c|c|c|}
\hline Variables & $\begin{array}{c}\text { Without EBV infection } \\
\mathbf{n}=\mathbf{3 6 7} \\
\mathbf{n}(\%)\end{array}$ & $\begin{array}{c}\text { With EBV infection } \\
\text { n=64 } \\
\text { n(\%) }\end{array}$ & $P$ value \\
\hline Age & & & 0.171 \\
\hline$<65 \mathrm{yrs}$ & $144(39.2)$ & $31(48.4)$ & \\
\hline$\geq 65 \mathrm{yrs}$ & $223(60.8)$ & $33(51.6)$ & \\
\hline Gender & & & 1.000 \\
\hline Male & $259(70.6)$ & $52(70.3)$ & \\
\hline Female & $108(29.4)$ & $17(29.7)$ & \\
\hline Tumor size & & & 1.000 \\
\hline$<5 \mathrm{~cm}$ & $141(38.4)$ & $25(39.1)$ & \\
\hline$\geq 5 \mathrm{~cm}$ & $226(61.6)$ & $39(60.9)$ & \\
\hline Tumor location & & & $<0.001$ \\
\hline Upper stomach & $55(15)$ & $25(39.1)$ & \\
\hline Middle stomach & $114(31.1)$ & $27(42.2)$ & \\
\hline Lower stomach & $185(50.4)$ & $12(18.8)$ & \\
\hline Whole stomach & $13(3.5)$ & 0 & \\
\hline Cell differentiation & & & 0.122 \\
\hline Poor & $199(56.4)$ & $35(54.7)$ & \\
\hline Moderate & $160(41.7)$ & $29(45.3)$ & \\
\hline Well & $8(1.9)$ & 0 & \\
\hline Gross appearance & & & 0.087 \\
\hline Superficial type & $50(13.6)$ & $6(9.4)$ & \\
\hline Borrmann type $1 \& 2$ & $95(25.9)$ & $25(39.4)$ & \\
\hline
\end{tabular}

(Continued) 


\begin{tabular}{|c|c|c|c|}
\hline Variables & $\begin{array}{c}\text { Without EBV infection } \\
\mathbf{n}=\mathbf{3 6 7} \\
\mathbf{n}(\%)\end{array}$ & $\begin{array}{c}\text { With EBV infection } \\
\begin{array}{c}\text { n=64 } \\
\text { n(\%) }\end{array}\end{array}$ & $P$ value \\
\hline Borrmann type $3 \& 4$ & $222(60.5)$ & $33(51.6)$ & \\
\hline Stromal reaction type & & & 0.850 \\
\hline Medullary type & $54(14.7)$ & $11(17.2)$ & \\
\hline Intermediate type & $195(53.1)$ & $34(53.1)$ & \\
\hline Scirrhous type & $118(32.2)$ & $19(29.7)$ & \\
\hline Lauren's histology & & & 1.000 \\
\hline Intestinal type & $200(54.5)$ & $35(54.7)$ & \\
\hline Diffuse type & $167(45.5)$ & $29(45.3)$ & \\
\hline Lymphovascular invasion & & & 0.097 \\
\hline Absent & $98(26.7)$ & $24(37.5)$ & \\
\hline Present & $269(73.3)$ & $40(62.5)$ & \\
\hline Pathological T category & & & 0.753 \\
\hline $\mathrm{T} 1$ & $59(16.1)$ & $8(12.5)$ & \\
\hline $\mathrm{T} 2$ & $57(15.5)$ & $12(18.8)$ & \\
\hline $\mathrm{T} 3$ & $124(33.8)$ & $24(37.5)$ & \\
\hline $\mathrm{T} 4$ & $127(34.6)$ & $20(31.3)$ & \\
\hline Pathological N category & & & 0.239 \\
\hline N0 & $122(33.2)$ & $17(26.6)$ & \\
\hline N1 & $56(15.3)$ & $15(23.4)$ & \\
\hline $\mathrm{N} 2$ & $97(26.4)$ & $13(20.3)$ & \\
\hline N3 & $92(25.1)$ & $19(29.7)$ & \\
\hline Pathological TNM Stage & & & 0.715 \\
\hline $\mathrm{I}$ & $78(21.3)$ & $11(17.2)$ & \\
\hline II & $102(27.8)$ & $20(31.3)$ & \\
\hline III & $187(51.0)$ & $33(51.6)$ & \\
\hline
\end{tabular}

Table 5: The association between genetic mutations in the PI3K/AKT pathway, PIK3CA amplifications and EBV infection in gastric cancer

\section{$\begin{array}{lll}\text { PIK3CA gene mutations } & \text { PIK3CA gene amplifications } & \text { PI3K/AKT pathway }\end{array}$} mutation

\begin{tabular}{|c|c|c|c|c|c|c|c|c|c|}
\hline & $\begin{array}{c}\text { Without } \\
\text { n=127 } \\
\text { n (\%) }\end{array}$ & $\begin{array}{l}\text { With } \\
\text { n=14 } \\
\text { n (\%) }\end{array}$ & $P$ value & $\begin{array}{c}\text { Without } \\
\text { n=87 } \\
\text { n (\%) }\end{array}$ & $\begin{array}{c}\text { With } \\
\text { n=109 } \\
\text { n (\%) }\end{array}$ & $P$ value & $\begin{array}{c}\text { Without } \\
\text { n=118 } \\
\text { n (\%) }\end{array}$ & $\begin{array}{l}\text { With } \\
\text { n=23 } \\
\text { n (\%) }\end{array}$ & $P$ value \\
\hline $\begin{array}{l}\text { EBV } \\
\text { infection }\end{array}$ & & & 0.001 & & & 0.689 & & & 0.046 \\
\hline Absent & $108(85)$ & $6(42.9)$ & & $73(83.9)$ & $\begin{array}{c}94 \\
(86.2)\end{array}$ & & $99(83.9)$ & $\begin{array}{c}15 \\
(65.2)\end{array}$ & \\
\hline Present & $19(15)$ & $8(57.1)$ & & $14(16.1)$ & $\begin{array}{c}15 \\
(13.8)\end{array}$ & & $19(16.1)$ & $8(34.8)$ & \\
\hline
\end{tabular}


Table 6: The initial recurrence pattern in intestinal-type gastric cancer patients after curative surgery

\begin{tabular}{|c|c|c|c|c|c|c|}
\hline & $\begin{array}{c}\text { PI3K/AKT } \\
\text { pathway } \\
\text { mutation } \\
(-) \\
\text { n=184 } \\
\text { n (\%) }\end{array}$ & $\begin{array}{c}\text { PI3K/ } \\
\text { AKT } \\
\text { pathway } \\
\text { mutation } \\
(+) \\
\mathbf{n}=\mathbf{5 1} \\
\mathbf{n}(\%)\end{array}$ & $P$ value & $\begin{array}{c}\text { PIK3CA } \\
\text { amplification } \\
(-) \\
\text { n=138 } \\
\text { n }(\%)\end{array}$ & $\begin{array}{c}\text { PIK3CA } \\
\text { amplification } \\
(+) \\
\text { n=97 } \\
\text { n }(\%)\end{array}$ & $P$ value \\
\hline Total patients with recurrence & $57(31.0)$ & $22(43.1)$ & 0.131 & $46(33.3)$ & $33(34)$ & 1.000 \\
\hline Locoregional recurrence & $20(10.9)$ & $11(21.6)$ & 0.060 & $20(14.5)$ & $12(12.4)$ & 0.702 \\
\hline Perigastric area & $5(2.7)$ & $2(3.9)$ & 0.647 & $4(2.9)$ & $3(3.1)$ & 1.000 \\
\hline Hepatoduodenal ligament & $16(8.7)$ & $8(15.7)$ & 0.188 & $14(10.1)$ & $10(10.3)$ & 1.000 \\
\hline Anastomosis & $5(2.7)$ & $2(3.9)$ & 0.647 & $5(3.6)$ & $2(2.1)$ & 0.703 \\
\hline Abdominal wall & $10(5.4)$ & $4(7.8)$ & 0.510 & $9(6.5)$ & $5(5.2)$ & 0.783 \\
\hline Duodenal stump & $1(0.5)$ & 0 & 1.000 & $1(0.7)$ & $1(1)$ & 1.000 \\
\hline Distant metastasis & $41(22.3)$ & $13(25.5)$ & 0.707 & $28(20.3)$ & $27(27.8)$ & 0.211 \\
\hline Peritoneal dissemination & $15(8.2)$ & $6(11.8)$ & 0.413 & $10(7.2)$ & $11(11.3)$ & 0.354 \\
\hline Hematogenous metastasis & $19(10.3)$ & $9(17.6)$ & 0.219 & $15(10.9)$ & $13(13.4)$ & 0.548 \\
\hline Liver & $18(9.8)$ & $9(17.6)$ & 0.137 & $15(10.9)$ & $12(12.4)$ & 0.836 \\
\hline Lung & $6(3.3)$ & $1(2.0)$ & 1.000 & $3(2.2)$ & $4(4.1)$ & 0.451 \\
\hline Bone & $5(2.7)$ & 0 & 0.588 & $3(2.2)$ & $2(2.1)$ & 1.000 \\
\hline Brain & $1(0.5)$ & 0 & 1.000 & 0 & $1(1)$ & 0.413 \\
\hline Distant lymphatic recurrence & $10(5.4)$ & $4(7.8)$ & 0.510 & $10(7.2)$ & $5(5.2)$ & 0.597 \\
\hline Virchow's node & $2(1.1)$ & $1(2.0)$ & 0.522 & $2(1.4)$ & $1(1)$ & 1.000 \\
\hline Para-aortic lymph node & $9(4.9)$ & $3(5.9)$ & 0.726 & $8(5.8)$ & $5(5.2)$ & 1.000 \\
\hline
\end{tabular}

Some patients had more than one recurrence pattern

\section{Initial recurrence patterns}

\section{Mutations in PI3K/AKT pathway genes}

A total of 154 patients (35.7\%) experienced tumor recurrence within a median period of 31.7 months (range: 0.47-183.0 months) after the initial follow-up visit. Patients with mutations in the PI3K/AKT pathway genes had more hematogenous metastases than did those without mutations $(18.8 \%$ vs. $7.5 \%$ for patients with vs. without mutations, respectively; $P=0.006$ ), particularly liver metastasis $(18.8 \%$ vs. $7.2 \%$ for patients with vs. without mutations, respectively; $P=0.005$ ). Regarding intestinaltype gastric cancer, no significant difference was observed between the recurrence patterns in patients with or without PI3K/AKT pathway gene mutations (Table 6). Regarding diffuse-type gastric cancer, patients with mutations in the PI3K/AKT pathway genes were more likely to have hematogenous metastases than were those without mutations $(22.2 \%$ vs. $4.5 \%$ for patients with vs. without mutations, respectively; $P=0.016$ ), particularly metastases in the liver and lungs (Table 7).

\section{PIK3CA amplification}

Among the 206 patients with PIK3CA amplifications, 34 patients $(16.5 \%)$ experienced peritoneal recurrence, which was significantly higher than the rate for those patients without PIK3CA amplifications (22/225, $9.8 \%, P=0.045)$. With regard to intestinal-type or diffusetype gastric cancer, however, there was no significant difference between the initial recurrence patterns in patients with or without PIK3CA amplifications. 
Table 7: The initial recurrence pattern in diffuse-type gastric cancer patients after curative surgery

\begin{tabular}{|c|c|c|c|c|c|c|}
\hline & $\begin{array}{c}\text { PI3K/ } \\
\text { AKT } \\
\text { pathway } \\
\text { mutation } \\
(-) \\
\text { n=178 } \\
\text { n (\%) }\end{array}$ & $\begin{array}{c}\text { PI3K/ } \\
\text { AKT } \\
\text { pathway } \\
\text { mutation } \\
(+) \\
\text { n=18 } \\
\text { n (\%) }\end{array}$ & $P$ value & $\begin{array}{c}P I K 3 C A \\
\text { amplification } \\
(-) \\
n=87 \\
\text { n }(\%)\end{array}$ & $\begin{array}{c}P I K 3 C A \\
\text { amplification } \\
(+) \\
\text { n=109 } \\
\text { n (\%) }\end{array}$ & $P$ value \\
\hline Total patients with recurrence & $68(38.2)$ & 7 (38.9) & 1.000 & $26(29.9)$ & $49(45)$ & 0.038 \\
\hline Locoregional recurrence & $12(6.7)$ & $3(16.7)$ & 0.146 & $7(8)$ & $8(7.3)$ & 1.000 \\
\hline Perigastric area & $2(1.1)$ & $1(5.6)$ & 0.252 & $2(2.3)$ & $1(0.9)$ & 0.586 \\
\hline Hepatoduodenal ligament & $7(3.9)$ & $3(16.7)$ & 0.052 & $4(4.6)$ & $6(5.5)$ & 1.000 \\
\hline Anastomosis & $6(3.4)$ & 0 & 1.000 & $4(4.6)$ & $2(1.8)$ & 0.409 \\
\hline Abdominal wall & $4(2.2)$ & $2(11.1)$ & 0.096 & $3(3.4)$ & $3(2.8)$ & 1.000 \\
\hline Distant metastasis & $45(25.3)$ & $7(38.9)$ & 0.257 & $20(23)$ & $32(29.4)$ & 0.334 \\
\hline Peritoneal dissemination & $31(17.4)$ & $4(22.2)$ & 0.693 & $12(13.8)$ & $23(21.1)$ & 0.196 \\
\hline Hematogenous metastasis & $8(4.5)$ & $4(22.2)$ & 0.016 & $6(6.9)$ & $6(5.5)$ & 0.769 \\
\hline Liver & $8(4.5)$ & $4(22.2)$ & 0.016 & $6(6.9)$ & $6(5.5)$ & 0.769 \\
\hline Lung & $2(1.1)$ & $2(11.1)$ & 0.043 & $3(3.4)$ & $1(0.9)$ & 0.324 \\
\hline Bone & $2(1.1)$ & $1(5.6)$ & 0.252 & $2(2.3)$ & $1(0.9)$ & 0.586 \\
\hline Brain & $1(0.6)$ & 0 & 1.000 & 0 & $1(0.9)$ & 1.000 \\
\hline Adrenal & $1(0.6)$ & 0 & 1.000 & 0 & $1(0.9)$ & 1.000 \\
\hline Skin & $1(0.6)$ & 0 & 1.000 & 0 & $1(0.9)$ & 1.000 \\
\hline Distant lymphatic recurrence & $16(9.0)$ & $1(5.6)$ & 1.000 & $7(8)$ & $10(9.2)$ & 1.000 \\
\hline Virchow's node & $2(1.1)$ & 0 & 1.000 & 0 & $2(1.8)$ & 0.504 \\
\hline Lymphangitic carcinomatosis & $1(0.6)$ & 0 & 1.000 & $1(1.1)$ & 0 & 0.444 \\
\hline Para-aortic lymph node & $14(7.9)$ & $1(5.6)$ & 1.000 & $6(6.9)$ & $9(8.3)$ & 0.792 \\
\hline
\end{tabular}

Some patients had more than one recurrence pattern

\section{Disease-free survival}

The 5-year disease-free survival rates of patients with or without mutations in the PI3K/AKT pathway genes were not significantly different $(35.9 \%$ vs. $47.2 \%$ for patients with vs. without mutations, respectively; $P=0.156$ ), and those of the two groups with either early gastric cancer (T1 tumors, $80 \%$ vs. $83.6 \%, P=0.800$ ) or advanced gastric cancer (T2-T4 tumors, $31.0 \%$ vs. $40.1 \%$, $P=0.235)$ were also not significantly different.

The 5-year disease-free survival rates of patients with or without $P I K 3 C A$ amplifications were similar ( $44.1 \%$ vs. $46.7 \%, P=0.367$ ), and those of the two groups with either early gastric cancer $(79.6 \%$ vs. $88.6 \%$, $P=0.588)$ or advanced gastric cancer $(37 \%$ vs. $40.2 \%$, $P=0.327$ ) were also note significantly different.
Univariate analysis showed that gender, tumor size, tumor location, gross appearance, stromal reaction type, and the pathological $\mathrm{T}$ and $\mathrm{N}$ categories were prognostic factors for disease-free survival (Table 8). Multivariate analysis showed that tumor size, tumor location, gross appearance, and pathological $\mathrm{T}$ and $\mathrm{N}$ categories were independent prognostic factors that affected disease-free survival (Table 9).

For patients with intestinal-type gastric cancer, the 5 -year disease-free survival rates of those with or without mutations in the PI3K/AKT pathway genes were not significantly different $(36.9 \%$ vs. $53.7 \%$ for those with vs. without mutations, respectively; $P=0.051$ ). For patients with diffuse-type gastric cancer, the 5-year disease-free survival rates of those with or without mutations in the $\mathrm{PI} 3 \mathrm{~K} / \mathrm{AKT}$ pathway genes were not significantly different 
Table 8: Univariate analysis of factors affecting survival of gastric cancer patients after curative surgery by the Kaplan-Meier method

\begin{tabular}{|c|c|c|c|c|c|c|}
\hline & \multicolumn{3}{|c|}{ Disease-free survival } & \multicolumn{3}{|c|}{ Overall survival } \\
\hline & HR & $95 \% \mathrm{CI}$ & $P$ value & HR & $95 \% \mathrm{CI}$ & $P$ value \\
\hline Age $(y / o)$ & & & 0.061 & & & 0.001 \\
\hline$<65$ & 1.00 & & & 1.00 & & \\
\hline$\geq 65$ & 1.30 & $0.988-1.703$ & & 1.65 & $1.229-2.206$ & \\
\hline Gender & & & 0.037 & & & 0.002 \\
\hline Male & 1.00 & & & 1.00 & & \\
\hline Female & 0.72 & $0.534-0.981$ & & 0.59 & $0.425-0.818$ & \\
\hline Tumor size $(\mathrm{cm})$ & & & $<0.001$ & & & $<0.001$ \\
\hline$<5$ & 1.00 & & & 1.00 & & \\
\hline$\geq 5$ & 2.52 & $1.859-3.417$ & & 2.54 & $1.839-3.499$ & \\
\hline Tumor location & & & $<0.001$ & & & $<0.001$ \\
\hline Upper third stomach & 1.00 & & & 1.00 & & \\
\hline Middle third stomach & 0.60 & $0.408-0.876$ & & 0.59 & $0.396-0.890$ & \\
\hline Lower third stomach & 0.76 & $0.539-1.064$ & & 0.84 & $0.595-1.193$ & \\
\hline Whole stomach & 2.38 & $1.196-4.746$ & & 2.46 & $1.238-4.902$ & \\
\hline Gross appearance & & & $<0.001$ & & & $<0.001$ \\
\hline Superficial type & 1.00 & & & 1.00 & & \\
\hline Borrmann type $1 \& 2$ & 2.53 & $1.426-4.503$ & & 2.67 & $1.471-4.861$ & \\
\hline Borrmann type $3 \& 4$ & 4.56 & $2.678-7.769$ & & 4.27 & $2.466-7.406$ & \\
\hline Cell differentiation & & & 0.333 & & & 0.368 \\
\hline Poor & 1.00 & & & 1.00 & & \\
\hline Moderate & 0.82 & $0.632-1.066$ & & 0.84 & $0.636-1.099$ & \\
\hline Well & 0.98 & $0.362-2.656$ & & 0.68 & $0.252-1.855$ & \\
\hline Lauren's classification & & & 0.484 & & & 0.738 \\
\hline Intestinal type & 1.00 & & & 1.00 & & \\
\hline Diffuse type & 1.10 & $0.847-1.418$ & & 1.05 & $0.798-1.374$ & \\
\hline Stromal Reaction type & & & 0.003 & & & 0.012 \\
\hline Medullary type & 1.00 & & & 1.00 & & \\
\hline Intermediate type & 1.59 & $1.042-2.437$ & & 1.61 & $1.048-2.472$ & \\
\hline Scirrhous type & 2.11 & $1.358-3.280$ & & 1.96 & $1.256-3.065$ & \\
\hline Pathological T category & & & $<0.001$ & & & $<0.001$ \\
\hline $\mathrm{T} 1$ & 1.00 & & & 1.00 & & \\
\hline $\mathrm{T} 2$ & 1.87 & $1.019-3.431$ & & 1.89 & $1.008-3.538$ & \\
\hline $\mathrm{T} 3$ & 2.83 & $1.666-4.819$ & & 2.62 & $1.504-4.560$ & \\
\hline $\mathrm{T} 4$ & 5.24 & $3.145-8.727$ & & 5.05 & $2.990-8.537$ & \\
\hline
\end{tabular}

(Continued) 


\begin{tabular}{|c|c|c|c|c|c|c|}
\hline & \multicolumn{3}{|c|}{ Disease-free survival } & \multicolumn{3}{|c|}{ Overall survival } \\
\hline & HR & $95 \% \mathrm{CI}$ & $P$ value & HR & $95 \% \mathrm{CI}$ & $P$ value \\
\hline Pathological N category & & & $<0.001$ & & & $<0.001$ \\
\hline No & 1.00 & & & 1.00 & & \\
\hline N1 & 1.14 & $0.725-1.799$ & & 1.08 & $0.666-1.743$ & \\
\hline $\mathrm{N} 2$ & 2.38 & $1.653-3.421$ & & 2.37 & $1.632-3.446$ & \\
\hline N3 & 4.84 & $3.369-6.940$ & & 5.56 & $3.814-8.113$ & \\
\hline $\begin{array}{l}\text { PI3K/AKT pathway } \\
\text { mutation }\end{array}$ & & & 0.156 & & & 0.529 \\
\hline Absent & 1.00 & & & 1.00 & & \\
\hline Present & 1.27 & $0.913-1.770$ & & 0.89 & $0.625-1.273$ & \\
\hline PIK3CA amplification & & & 0.103 & & & 0.368 \\
\hline Absent & 1.00 & & & 1.00 & & \\
\hline Present & 1.25 & $0.956-1.634$ & & 1.13 & $0.871-1.453$ & \\
\hline
\end{tabular}

( $30.0 \%$ vs. $40.3 \%$ for patients with vs. without mutations, respectively; $P=0.897$ ).

For patients with intestinal-type gastric cancer, the 5-year disease-free survival rates of those with or without PIK3CA amplifications were not significantly different ( $49.8 \%$ vs. $50.4 \%$ for those with vs. without mutations, respectively; $P=0.533$ ). For patients with diffuse-type gastric cancer, the 5-year disease-free survival rates of those with or without PIK3CA amplifications were not significantly different $(38.9 \%$ vs. $38.5 \%$ for patients with vs. without mutations, respectively; $P=0.573$ ).

\section{Overall survival}

\section{Mutations in PI3K/AKT pathway genes}

The 5-year overall survival rates of patients with or without mutations in the PI3K/AKT pathway genes were also not significantly different $(55.5 \%$ vs. $56.4 \%$ $P=0.529$ ), and those of the two groups of either early gastric cancer $(83.3 \%$ vs. $88.6 \%, P=0.977)$ or advanced gastric cancer $(51.4 \%$ vs. $49.2 \%, P=0.303)$ were also not significantly different.

The 5-year overall survival rates of patients with or without $P I K 3 C A$ amplifications were not significantly different ( $54.4 \%$ vs. $57.1 \%, P=0.102)$, and those of the two groups with either early gastric cancer $(85.4 \%$ vs. $91.3 \%, P=0.662$ ) or advanced gastric cancer were also not significantly different $(47.3 \%$ vs. $51.5 \%, P=0.055)$.

Univariate analysis showed that age, gender, tumor size, tumor location, gross appearance, stromal reaction type, and the pathological $\mathrm{T}$ category and $\mathrm{N}$ category were prognostic factors for overall survival (Table 8). Multivariate analysis showed that age, gross appearance, and the pathological $\mathrm{T}$ and $\mathrm{N}$ categories were independent prognostic factors that affected overall survival (Table 9).

Regarding patients with intestinal-type gastric cancer, the 5-year overall survival rates of patients with or without mutations in the PI3K/AKT pathway genes were not significantly different $(57.7 \%$ vs. $55.5 \%$ for patients with vs. without mutations, respectively; $P=0.851$ ). For those with diffuse-type gastric cancer, the 5 -year overall survival rates of patients with or without mutations in the PI3K/AKT pathway genes were not significantly different ( $55.5 \%$ vs. $53.6 \%$ for patients with vs. without mutations, respectively; $P=0.440$ ).

Regarding patients with intestinal-type gastric cancer, the 5-year overall survival rates of those with or without PIK3CA amplifications were not significantly different (54.3\% vs. $58.8 \%$ for patients with vs. without mutations, respectively; $P=0.103$ ). Regarding patients with diffuse-type gastric cancer, the 5-year overall survival rates of those with or without PIK3CA amplifications were not significantly different $(54.5 \%$ vs. $54.1 \%$ for patients with vs. without mutations, respectively; $P=0.542$ ).

\section{DISCUSSION}

This study presents the genetic profiles of PI3K/ AKT pathway components and the association of genetic mutations in this pathway with the clinicopathological characteristics, initial recurrence patterns and prognoses of gastric cancer patients. Our novel findings showed that intestinal-type gastric cancer with $P I 3 K / A K T$ pathway mutations was associated with tumors in the lower-third of the stomach, whereas diffuse-type gastric cancer with $\mathrm{PI} 3 \mathrm{~K} / \mathrm{AKT}$ pathway mutations was associated with tumors 
Table 9: Multivariate Cox proportional-hazards model using the forward logistics regression stepwise procedure for the analysis of the survival of the gastric cancer patients after curative surgery

\begin{tabular}{|c|c|c|c|c|c|c|}
\hline & \multicolumn{3}{|c|}{ Disease-free survival } & \multicolumn{3}{|c|}{ Overall survival } \\
\hline & HR & $95 \% \mathrm{CI}$ & $P$ value & HR & $95 \% \mathrm{CI}$ & $P$ value \\
\hline Age $(y / o)$ & & & & & & 0.001 \\
\hline$<65$ & & & & 1.00 & & \\
\hline$\geq 65$ & & & & 1.78 & $1.318-2.393$ & \\
\hline Tumor size $(\mathrm{cm})$ & & & 0.013 & & & \\
\hline$<5$ & 1.00 & & & & & \\
\hline$\geq 5$ & 1.54 & $1.093-2.155$ & & & & \\
\hline Tumor location & & & 0.039 & & & \\
\hline Upper third stomach & 1.00 & & & & & \\
\hline Middle third stomach & 0.68 & $0.459-1.013$ & & & & \\
\hline Lower third stomach & 0.63 & 0.439-0.889 & & & & \\
\hline Whole stomach & 1.05 & $0.515-2.137$ & & & & \\
\hline Gross appearance & & & 0.006 & & & 0.007 \\
\hline Superficial type & 1.00 & & & 1.00 & & \\
\hline Borrmann type $1 \& 2$ & 1.55 & $0.782-3.073$ & & 1.67 & $0.829-3.368$ & \\
\hline Borrmann type $3 \& 4$ & 2.36 & $1.199-4.628$ & & 2.47 & $1.244-4.919$ & \\
\hline Pathological T category & & & 0.044 & & & $<0.001$ \\
\hline $\mathrm{T} 1$ & 1.00 & & & 1.00 & & \\
\hline $\mathrm{T} 2$ & 1.10 & $0.551-2.213$ & & 0.82 & $0.396-1.698$ & \\
\hline $\mathrm{T} 3$ & 1.15 & $0.584-2.258$ & & 0.98 & $0.487-1.978$ & \\
\hline $\mathrm{T} 4$ & 1.71 & $0.857-3.392$ & & 1.71 & $0.867-3.378$ & \\
\hline Pathological N category & & & $<0.001$ & & & $<0.001$ \\
\hline N0 & 1.00 & & & 1.00 & & \\
\hline N1 & 0.83 & $0.518-1.321$ & & 0.89 & $0.543-1.467$ & \\
\hline N2 & 1.48 & $0.997-2.187$ & & 1.71 & $1.150-2.556$ & \\
\hline N3 & 2.69 & $1.797-4.019$ & & 4.15 & $2.758-6.242$ & \\
\hline
\end{tabular}

in the upper-third of the stomach; diffuse-type gastric cancer with $\mathrm{PI} 3 \mathrm{~K} / \mathrm{AKT}$ pathway mutations was also associated with more hematogenous metastasis. Mutations in PI3K/AKT pathway genes were not independent risk factors for the overall survival or disease-free survival of either intestinal-type and diffuse-type gastric cancer patients.

As previously reported [19], the authors proposed a molecular classification dividing gastric cancer into four subtypes, including EBV positive, chromosomal instability, microsatellite instability, and genomically stable. Chromosomal instability (including intestinal histology, TP53 mutation, RTK-RAS activation) was more frequently located in the fundus \& body (38.8\%), followed by the antrum (33.3\%) and cardia $(25.2 \%)$; enrichment of the diffuse histology was observed in the genomically stable group which was more frequently located in the antrum $(48.3 \%)$, followed by fundus \& body (31.0\%) and cardia (19.0\%). A previous study [24] showed that the PI3K/AKT pathway plays an important role in the development of diffuse-type gastric cancer in the upper stomach and the esophagogastric junction, which is consistent with our findings. Consequently, a survey of $\mathrm{PI} 3 \mathrm{~K} / \mathrm{AKT}$ pathway mutations is required for patients 
with a diffuse-type gastric cancer in the upper stomach and might be helpful for choosing a targeted therapy. Furthermore, our novel findings revealed that in the case of intestinal-type gastric cancer, patients with PI3K/AKT pathway mutations were more likely to have tumors located in the lower-third of the stomach. It is interesting that the distribution of gastric cancer was correlated with the histological type and with the presence of PI3K/AKT pathway mutations. More patients enrolled in the future are required to investigate these interesting findings.

A previous study [25] revealed that blockade of the PI3K/AKT pathway could inhibit liver metastasis of colorectal cancer. An in vivo study [26] showed that inhibition of the PI3K/AKT pathway could decrease the progression of liver metastases of pancreatic neuroendocrine tumors. MiR-7 was reported to function as a tumor suppressor and plays a substantial role in inhibiting the tumorigenesis and reversing the metastasis of hepatocellular carcinoma through the PI3K/AKT/ mTOR-signaling pathway both in vitro and in vivo [27]. Hence, the level of PI3K/AKT pathway activity might be correlated with the hematogenous metastasis of cancers. However, a correlation between PI3K/AKT pathway mutations and the distant metastasis of gastric cancer has not yet been reported. Our novel findings showed that patients with diffuse-type gastric cancer (but not other types of cancers) and PI3K/AKT pathway mutations were more likely to have liver and lung metastases compared with those without such mutations. Therefore, for patients with diffuse-type gastric cancer with liver metastasis, a survey of PI3K/AKT pathway mutations is recommended because targeted therapy might be helpful for patients with PI3K/AKT pathway mutations.

In this study, the mutation rate of $P I K 3 C A$ was $13.2 \%$ and that of PTEN was $4.0 \%$, which is in accordance with the reported mutation rate of $P I K 3 C A$ in gastric cancer [14] but is lower than the mutation rates (18.75\%) observed for PTEN in the study of Wen et. al. [28], who performed direct sequencing of all 9 exons of PTEN. Although the coverage rate of our MassARRAY for $P I K 3 C A$ mutations was $75.3 \%$ that of the COSMIC database, the coverage rate for PTEN mutations was only $25.2 \%$ (Figure 1). Variations in the detection methods used and in the patient populations may contribute to the lower incidence of PTEN mutations observed in the present study.

Some previous reports have suggested that patients with breast cancer who have PIK3CA mutations, have a better prognosis than others [29], whereas other authors have suggested that $P I K 3 C A$ mutations are associated with a worse prognosis in colorectal cancer, endometrial cancer and lung cancer patients [30-32]. No reports investigating the correlation between PI3K/AKT pathway mutations and patient survival after curative resection of gastric cancer have been published. Only Takahashi et al. [18] reported that PIK3CA mutations were not associated with the prognosis of patients with metastatic gastric cancer who were being treated with systemic chemotherapy. In their study, only PIK3CA mutations (rather than PI3K/AKT pathway mutations) were examined, and the incidence of PIK3CA mutations in gastric cancer patients was only 9 of 163 patients $(5.5 \%)$. To date, the current study is the only to investigate the effect of mutations in PI3K/ AKT pathway genes on the survival of a large population of patients with gastric cancer after curative surgery. Although no significant difference was observed in terms of the disease-free survival and overall survival of patients with or without PI3K/AKT pathway mutations, our results might help guide future studies of the PI3K/AKT pathway in gastric cancer.

Our results showed that only when the tumors were located in the middle-third of the stomach were more tumors with mutations in the PIK3CA gene or mutations in PI3K/AKT pathway genes were associated with an EBV infection than were those without such mutations. It is interesting that the correlation between mutations and EBV infection was observed only in the middle-third of the stomach, but not in the upper-third or lower-third of the stomach. The previous study [19] demonstrated that EBV-infected gastric cancer was associated with $P I K 3 C A$ mutation, particularly those occurring in the body of the stomach, which was similar to our findings. There are two possible reasons for the above findings. The first reason is that the EBV infection is higher in the middlethird of the stomach, causing the correlation between EBV infection and $P I K 3 C A$ mutations to be higher in this location of stomach. The second reason is that an EBV infection and PIK3CA mutations are more likely to induce carcinogenesis in the middle-third of the stomach. Our results showed that tumors with $P I K 3 C A$ mutations were more likely to be located in the lower-third of the stomach, whereas EBV infections were more frequent in the middle-third of the stomach. Therefore, the second reason might more likely explain our findings. However, various confounding factors, such as selection bias, racial differences, the sex ratio, and the complicated pathways involved in gastric carcinogenesis might have affected the results. Future large-scale studies investigating the correlation between EBV infection, PIK3CA mutations and tumor locations are required to address these interesting findings.

The Helicobacter pylori (H. pylori) VacA toxin was reported to activate the $\mathrm{PI} 3 \mathrm{~K} / \mathrm{AKT}$ pathway, resulting in the phosphorylation and the inhibition of GSK3 $\beta$ activity and the subsequent translocation of $\beta$-catenin to the nucleus, which is consistent with the effects of VacA on $\beta$-catenin-regulated transcriptional activity [33]. Thus, the PI3K/AKT pathway might play a role in pathogenesis resulting from an $H$. pylori infection, including gastric carcinogenesis. Whether an $H$. pylori infection is associated with mutations in PI3K/AKT pathway genes is unknown. Furthermore, information regarding an $H$. pylori 
infection is not available for all of our patients. Our future study will investigate the correlation between an $\mathrm{H}$. pylori infection and mutations in PI3K/AKT pathway genes.

Regarding PIK3CA amplifications in gastric cancer, our results showed that patients with PIK3CA amplifications were more likely to have diffuse-type, poorly differentiated gastric cancer and peritoneal seeding compared with patients without PIK3CA amplifications. However, the 5-year overall survival and disease-free survival of patients with or without PIK3CA amplifications were not significantly different. Regarding intestinal-type or diffuse-type gastric cancer, PIK3CA amplifications was not associated with the initial recurrence pattern or with survival. Whether PIK3CA amplifications are associated with the prognosis of gastric cancer remains controversial. In the patient series that Shi et al. investigated [11], patients with PIK3CA amplifications were found to have with a worse prognosis were than those without PIK3CA amplifications; however, Lee et al. [22] reported that PIK3CA amplifications were not associated with a worse prognosis. Lee et al. [22] proposed that the possible reason for this discrepancy might be the different proportions of patients with early gastric cancer (more than $40 \%$ of the patients in their series compared with only $14 \%$ of the patients in the series of Shi et al. [11]). However, the percentage of patients with early gastric cancer in our series was $15.5 \%$, which was similar to that in the series of Shi et al. Our data demonstrated no significantly difference in survival of patients with or without PIK3CA amplifications. Additionally, in the present study, no difference in the survival of patients with or without PIK3CA amplifications who had early or advanced gastric cancer was found. Consequently, the difference between the survival observed in the study of Shi et al. and that of Lee et al. is unlikely to be due to a difference in the percentage of patients with early gastric cancer. The reliability of clinical data is highly important for this type of study. Various confounding factors might affect the results. In our hospital, the gastric cancer patients were operated upon by experienced surgeons and the pathological review was performed by only one pathologist. Because our hospital is one of the best medical centers in northern Taiwan and more than $60 \%$ of our patients are veterans, more than $90 \%$ of our patients received postoperative treatment and follow-up in our hospital. We also created the only gastric cancer patient care group in Taiwan more than 20 years ago. We believe that our results are reliable. Based on our data, we conclude that PIK3CA amplifications are not associated with the prognosis of gastric cancer.

One of our novel findings is that PIK3CA amplifications were associated with more peritoneal recurrences of gastric cancers. This result is interesting and has not been previously reported. Further in vivo and intro studies investigating the possible mechanisms underlying the occurrence are required to substantiate our findings.
Moreover, physicians should be alert to peritoneal recurrence during the follow-up period, particularly in gastric cancer patients with $P I K 3 C A$ amplifications.

An inverse correlation between PIK3CA mutations and PIK3CA amplifications was reported in various cancers, including gastric cancer [11,34,35]. In our study, among those patients with PIK3CA mutations, none had PIK3CA amplifications and there were no PIK3CA mutations in the patients with $P I K 3 C A$ amplifications. These results suggest that different pathways are involved in PIK3CA mutations and amplifications in gastric cancer.

As shown in Figure 1(B), based on comparing the MassARRAY data with that om the COSMIC database, the coverage rate of the selected mutation hotspots in five genes analyzed that are involved in the PI3K/AKT pathway was $75.3 \%$ for PIK $3 C A, 98.2 \%$ for $A K T 1$, $53.9 \%$ for $A K T 2,64.2 \%$ for $A K T 3$, and $25.2 \%$ for PTEN. However, not all of the reported somatic alterations, e.g., point mutations, small insertions and deletions, large insertions and deletions, and gene fusion mutations in these genes were examined in this study. The most frequent mutations determined using the COSMIC database and found in previous studies provide a possible target list for investigating their clinical impact via translational genomics using a large sample size, such as that used in this study. Using multiple technologies and multiple types of materials (DNA, RNA, miRNA and protein) is an ideal strategy for comprehensively investigating the pathogenesis of gastric cancer; however, the purpose of the present study was to identify frequent clinically actionable DNA mutations to analyze in clinical samples obtained in the future. In addition, we considered each individual mutation site but not all of the mutations in each of the selected gene, so that sufficient data regarding only the mutation hotspots was obtained using the clinical samples and might have statistical significances.

A limitation of this study was that the mutation screening was limited to certain hotspots within five notable genes involved in the PI3K/AKT downstream pathway, which may have limited the power to detect significant clinicopathological and molecular correlations. In addition, we examined genetic mutations in our study rather than the loss of expression of the relevant encoded proteins, which might have led to underestimation of the actual frequency of genetic inactivation. Furthermore, not all of the patients with gastric cancer who had undergone surgery in our hospital were enrolled in this study. Therefore, selection bias might have occurred. The number of patients with diffuse-type gastric cancer with hematogenous metastasis was limited. Whereas the conclusions drawn might not be reliable, the statistical value of the data obtained is considerable. Nevertheless, our cohort is the largest to date in which the clinicpathological characteristics and recurrence patterns regarding mutations in PI3K/AKT pathway genes and PIK3CA amplifications in gastric cancers were 
investigated. More patients must be enrolled in the future to verify our current conclusions.

In conclusion, our findings provide evidence that mutations in PI3K/AKT pathway-related genes in diffusetype gastric cancers can affect the patterns of recurrence. Gastric cancer patients with PIK3CA amplifications had more diffuse-type and poorly differentiated gastric cancer and more peritoneal recurrences compared with patients without PIK3CA amplifications. Physicians should be aware of the possibility of hematogenous metastasis during the follow-up of patients with gastric cancer who have PI3K/AKT pathway mutations, particularly those with diffuse-type gastric cancer. During the follow-up of gastric cancer patients with $P I K 3 C A$ amplifications, physicians should be aware of the possibility of peritoneal recurrence.

\section{METERIALS AND METHODS}

Between October 2000 and March 2007, 431 patients with gastric cancer who had undergone curative resection were enrolled in this study, which was approved by the Institutional Review Board of the Taipei Veterans General Hospital. Informed consent was obtained and the procedures followed were in accordance with the ethical standards of the committee overseeing human experimentation at the Taipei Veterans General Hospital and the Helsinki Declaration of 1975, as revised in 1983. Patients with a history of gastric surgery or a pathological diagnosis other than adenocarcinoma were excluded. Patients who experienced major surgical complications or surgical mortality were also excluded.

The pathological staging of the gastric cancers was performed according to the seventh AJCC/UICC TNM classification system [36]. All of the surgeries were performed by surgeons who specialize in gastric cancer. The data were prospectively collected and were entered into a computer file, and the follow-up conditions of the patients were regularly updated.

Prior to surgery, all of the patients underwent chest radiography, abdominal sonography, or CT scanning for tumor staging. A total or distal subtotal gastrectomy was performed depending on the distance between the cardia and the tumor with a margin of $3 \mathrm{~cm}$ needed for superficial and well-defined tumors, and a margin of $5 \mathrm{~cm}$ needed for advanced or poorly defined tumors. A subtotal gastrectomy is the standard surgical procedure for distal gastric cancer, whereas a total gastrectomy is the more common surgical procedure for proximal gastric cancer.

\section{Follow-up}

The overall survival was calculated from the time of surgery until death or the date of the last follow-up visit. None of the patients received preoperative chemotherapy. Before 2008, adjuvant chemotherapy or radiotherapy after curative surgery was not routinely given; rather, such therapies were given only when tumor recurrence was diagnosed or highly suspected. Since 2008, adjuvant therapy (such as S-1) has been prescribed for stage II or stage III disease in our hospital after curative surgery due to its proven survival benefit [37]. None of the 431 patients enrolled in this study received adjuvant therapy after surgery.

Follow-up assessments were performed every 3 months for the first 5 years after surgery and every 6 months thereafter until the patient's death. The followup procedures involved medical histories, physical examinations, routine blood tests, liver function tests, measurement of the levels of tumor markers (e.g., carcinoembryonic antigen and carbohydrate antigen 199), chest radiography, abdominal sonography, and CT scanning.

\section{Detection of mutations using a highly sensitive MALDI-TOF technology}

Patients were considered to carry a PI3K/AKT pathway mutation when any one of the PIK3CA, PTEN, $A K T 1, A K T 2$, or $A K T 3$ genes contained a mutation. A total of 39 somatic mutations within the PI3K/AKT pathway genes were selected based on the frequency of their occurrence in tumor tissue according to the 'Catalogue of Somatic Mutations in Cancer' database [http:// cancer.sanger.ac.uk/cancergenome/projects/cosmic/] (Supplementary Table 1). Mutation assays were performed using a Sequenom MassARRAY system (Sequenom, San Diego, CA, USA). The PCR and single-base extension primers were designed using MassARRAY Assay Design 3.1 software, and three multiplex reactions were designed to detect these $39 \mathrm{PI} 3 \mathrm{~K} / \mathrm{AKT}$ pathway-related mutations. PCR reactions were performed in a final volume of $5 \mu \mathrm{l}$ containing $1 \mathrm{pmol}$ of the appropriate primers, $10 \mathrm{ng}$ of genomic DNA, and reaction mix (Sequenom) in 384well plates. The conditions for the PCR reactions, which were performed using tumor DNA to amplify the regions that harbored loci of interest, were as follows: $94^{\circ} \mathrm{C}$ for $15 \mathrm{~min}$, followed by 40 cycles at $94^{\circ} \mathrm{C}(20 \mathrm{~s}), 56^{\circ} \mathrm{C}(30$ $\mathrm{s})$, and $72^{\circ} \mathrm{C}(60 \mathrm{~s})$, with a final extension at $72^{\circ} \mathrm{C}$ for 3 min. During the primer extension step, the PCR products were incubated with probes that immediately annealed adjacent to the mutation sites and single-base extension was conducted in the presence of chain-terminating dideoxynucleotides that generated allele-specific DNA products. The single-base extension conditions were as follows: $94^{\circ} \mathrm{C}$ for $2 \mathrm{~min}$, followed by 40 cycles at $94^{\circ} \mathrm{C}(5 \mathrm{~s}), 52^{\circ} \mathrm{C}(5 \mathrm{~s})$, and $72^{\circ} \mathrm{C}(5 \mathrm{~s})$. The extension products were spotted onto a SpectroCHIP II (Sequenom) and were then analyzed using matrix-assisted laser desorption ionization-time of flight (MALDI-TOF) mass spectrometry (Sequenom) to determine the mutational status based on the difference in the mass of the mutant 
and wild-type bases. Each spectrum was then analyzed using Typer 4.0 software (Sequenom) to perform variant genotype calling. Putative mutations were further filtered by manual review. To estimate the percentage of a mutant allele, the relative signal intensity was determined using the following equation: (mutant peak area)/(mutant peak area+ wild-type peak area). The sensitivity of the MALDITOF mutational assay varied because a specific assay was designed for each individual mutation. To generate different dilutions of each mutation, we obtained wildtype and mutant dsDNA (gBlocks gene fragments) from Integrated DNA Technologies (Coralville, IA, USA) and used the qPCR method to quantify the number of DNA copies in each gBlock DNA fragment for pooling. The sensitivity of the assay for each of the PI3K/AKT pathway mutations considered in this study was as low as $1-5 \%$ (Supplementary Table 3 ); cluster plots and representative spectra of selected dilutions are shown in Supplementary Figure 1.

\section{PIK3CA amplifications}

The copy number of the $P I K 3 C A$ gene was analyzed using qPCR, with the LINE1 element used as an internal reference target, using the primer employed in a previous study [38]. qPCR was then conducted on an LightCycler 480 II system (Roche Diagnostics, Mannheim, Germany) in a total volume of $20 \mu \mathrm{l}$ of reaction mixture containing $10 \mu$ of KAPA SYBR FAST qPCR Master Mix (Kapa Biosystems, Woburn, MA, USA), 8 pmol of each primer, and $10 \mathrm{ng}$ of DNA. The PCR conditions were as follows: $95^{\circ} \mathrm{C}$ for $1 \mathrm{~min}$, followed by 40 cycles of $95^{\circ} \mathrm{C}$ for 15 $\mathrm{s}$, and $60^{\circ} \mathrm{C}$ for $30 \mathrm{~s}$. The threshold cycle number $(\mathrm{Ct})$ values of the PIK3CA gene and LINE1 were determined. The PIK3CA gene copy number was calculated using the delta-delta-Ct method in triplicate [39]. The relative copy number in each sample was determined by comparing the ratio of $P I K 3 C A$ gene to the LINE1 element in each sample, and 20 normal human genomic DNA samples isolated from human blood cells were used to generate the diploid delta-Ct distribution. As previously reported [11], amplification of the $P I K 3 C A$ gene was defined as a copy number of $\geq 3$ with $P$-value of $<0.05$.

\section{Statistical analysis}

The statistical analyses were performed using SPSS software (version 16.0 for Windows, SPSS, Chicago, IL, USA). All of the results in the text and tables are presented as the means values \pm the standard deviations (SD). The categorical data were compared using a $\chi 2$ test with Yates correction or Fisher's exact test. The overall survival was measured from the date of the operation to the date of death or the final follow-up. The disease-free survival was defined as the length of time after gastric cancer surgery during which the patient survived without tumor recurrence. The distributions of overall survival and disease-free survival were estimated using the Kaplan-Meier method. Cox proportional hazards models were used to explore the association between the clinical parameters and the overall survival and disease-free survival. A $P$ value of $<0.05$ was considered statistically significant.

\section{ACKNOWLEDGMENTS}

The authors thank Dr. Chien-Hsing Lin for technical support and analysis.

\section{CONFLICTS OF INTEREST}

The authors declare no conflicts of interest.

\section{GRANT SUPPORT}

This study was supported by research grants from the Taipei Veterans General Hospital (V104C-071), the Szu-Yuan Research Foundation of Internal Medicine (Number: 103024), and the Ministry of Science and Technology (100-2314-B-075-011-MY3, 102-2314-B075-065, 103-2314-B-075-042).

\section{REFERENCES}

1. Parkin DM, Bray F, Ferlay J, Pisani P. Global cancer statistics, 2002. CA Cancer J Clin 2005;55:74-108.

2. Wu CW, Lo SS, Shen KH, Hsieh MC, Chen JH, Chiang JH, $\mathrm{Lin} \mathrm{HJ}$, et al. Incidence and factors associated with recurrence patterns after intended curative surgery for gastric cancer. World J Surg 2003;27:153-158.

3. Deng N, Goh LK, Wang H, Das K, Tao J, Tan IB, Zhang $\mathrm{S}$, et al. A comprehensive survey of genomic alterations in gastric cancer reveals systematic patterns of molecular exclusivity and co-occurrence among distinct therapeutic targets. Gut 2012;61:673-684.

4. Wadhwa R, Song S, Lee JS, Yao Y, Wei Q, Ajani JA. Gastric cancer-molecular and clinical dimensions. Nat Rev Clin. Oncol 2013;10:643-655.

5. Willems L, Tamburini J, Chapuis N, Lacombe C, Mayeux P, Bouscary D. PI3K and mTOR signaling pathways in cancer: New data on targeted therapies. Curr. Oncol. Rep 2012;14:129-138.

6. Fresno Vara JA, Casado E, de Castro J, Cejas P, BeldaIniesta C, González-Barón M. PI3K/Akt signalling pathway and cancer. Cancer Treat Rev 2004;30:193-204.

7. Zhou J, Chen GB, Tang YC, Sinha RA, Wu Y, Yap CS, Wang $G$, et al. Genetic and bioinformatic analyses of the expression and function of PI3K regulatory subunit PIK3R3 in an Asian patient gastric cancer library. BMC Med Genomics 2012;5:34. 
8. Tapia O, Riquelme I, Leal P, Sandoval A, Aedo S, Weber $\mathrm{H}$, Letelier $\mathrm{P}$, et al. The PI3K/AKT/mTOR pathway is activated in gastric cancer with potential prognostic and predictive significance. Virchows Arch. 2014;465:25-33

9. Ying J, Xu Q, Liu B, Zhang G, Chen L, Pan H. The expression of the $\mathrm{PI} 3 \mathrm{~K} / \mathrm{AKT} / \mathrm{mTOR}$ pathway in gastric cancer and its role in gastric cancer prognosis. Onco Targets Ther. 2015;8:2427-33.

10. Riquelme I, Saavedra K, Espinoza JA, Weber H, García P, Nervi B, Garrido M, et al. Molecular classification of gastric cancer: Towards a pathway-driven targeted therapy. Oncotarget. 2015;6:24750-24779. doi: 10.18632/ oncotarget.4990.

11. Shi J, Yao D, Liu W, Wang N, Lv H, Zhang G, Ji M, et al. Highly frequent PIK3CA amplification is associated with poor prognosis in gastric cancer. BMC Cancer. 2012;12:50.

12. Siegfried Z, Bonomi S, Ghigna C, Karni R. Regulation of the Ras-MAPK and PI3K-mTOR signaling pathways by alternative splicing in cancer. Int J Cell Biol. 2013; 2013:568931.

13. Samuels Y, Wang Z, Bardelli A, Silliman N, Ptak J, Szabo $\mathrm{S}$, Yan $\mathrm{H}$, et al. High frequency of mutations of the PIK3CA gene in human cancers. Science 2004;304:554.

14. Velho S, Oliveira C, Ferreira A, Ferreira AC, Suriano G, Schwartz S Jr, Duval A, et al. The prevalence of PIK3CA mutations in gastric and colon cancer. Eur $\mathrm{J}$ Cancer 2005;41:1649-1654.

15. Barbi S, Cataldo I, de Manzoni G, Bersani S, Lamba S, Mattuzzi S, Bardelli A, et al. The analysis of PIK3CA mutations in gastric carcinoma and metanalysis of literature suggest that exon-selectivity is a signature of cancer type. J Exp Clin Cancer Res 2010;29:32.

16. Sukawa $\mathrm{Y}$, Yamamoto $\mathrm{H}$, Nosho, K, Ito M, Igarashi $\mathrm{H}$, Naito T, Mitsuhashi $\mathrm{K}$, et al. HER2 expression and PI3K-Akt pathway alterations in gastric cancer. Digestion 2014;89:12-17.

17. Corso G, Velho S, Paredes J, Pedrazzani C, Martins D, Milanezi F, Pascale V, et al. Oncogenic mutations in gastric cancer with microsatellite instability. Eur J Cancer 2011;47:443-451.

18. Takahashi N, Yamada Y, Taniguchi H, Fukahori M, Sasaki Y, Shoji H, Honma Y, et al. Clinicopathological features and prognostic roles of KRAS, BRAF, PIK3CA and NRAS mutations in advanced gastric cancer. BMC Res Notes 2014;29;7:271.

19. Cancer Genome Atlas Research Network. Comprehensive molecular characterization of gastric adenocarcinoma. Nature. 2014;513:202-9.

20. Lee J, van Hummelen P, Go C, Palescandolo E, Jang J, Park HY, Kang SY, et al. High-throughput mutation profiling identifies frequent somatic mutations in advanced gastric adenocarcinoma. PLoS One. 2012;7:e38892.

21. Yang Q, Shao Y, Shi J, Qu Y, Wu K, Dang S, Shi B, et al. Concomitant PIK3CA amplification and RASSF1A or
PAX6 hypermethylation predict worse survival in gastric cancer. Clin Biochem. 2014;47:111-6.

22. Lee H, Hwang IS, Choi IJ, Kang YN, Park KU, Lee JH. Are PIK3CA Mutation and Amplification Associated with Clinicopathological Characteristics of Gastric Cancer? Asian Pac J Cancer Prev. 2015;16:4493-6.

23. Tran TN, Brettingham-Moore K, Duong CP, Mitchell C, Clemons NJ, Phillips WA. Molecular changes in the phosphatidylinositide 3-kinase (PI3K) pathway are common in gastric cancer. J Surg Oncol. 2013;108:113-20.

24. Yamamoto H, Watanabe Y, Maehata T, Morita R, Yoshida Y, Oikawa R, Ishigooka S, et al. An updated review of gastric cancer in the next-generation sequencing era: Insights from bench to bedside and vice versa. World J Gastroenterol 2014;20:3927-3937.

25. Yuge R, Kitadai Y, Shinagawa K, Onoyama M, Tanaka S, Yasui W, Chayama K. mTOR and PDGF Pathway Blockade Inhibits Liver Metastasis of Colorectal Cancer by Modulating the Tumor Microenvironment. Am J Pathol 2015;185:399-408.

26. Djukom C, Porro LJ, Mrazek A, Townsend CM Jr, Hellmich MR, Chao C. Dual inhibition of PI3K and mTOR signaling pathways decreases human pancreatic neuroendocrine tumor metastatic progression. Pancreas 2014;43:88-92.

27. Fang Y, Xue JL, Shen Q, Chen J, Tian L. MicroRNA-7 inhibits tumor growth and metastasis by targeting the phosphoinositide 3-kinase/Akt pathway in hepatocellular carcinoma. Hepatology 2012;55:1852-1862.

28. Wen YG, Wang Q, Zhou CZ, Qiu GQ, Peng ZH, Tang HM. Mutation analysis of tumor suppressor gene PTEN in patients with gastric carcinomas and its impact on PI3K/ AKT pathway. Oncol Rep 2010;24:89-95.

29. Kalinsky K, Jacks LM, Heguy A, Patil S, Drobnjak M, Bhanot UK, Hedvat CV, et al. PIK3CA mutation associates with improved outcome in breast cancer. Clin Cancer Res 2009, 15:5049-5059.

30. Mao C, Yang ZY, Hu XF, Chen Q, Tang JL. PIK3CA exon 20 mutations as a potential biomarker for resistance to anti-EGFR monoclonal antibodies in KRAS wild-type metastatic colorectal cancer: a systematic review and metaanalysis. Ann Oncol 2012, 23:1518-1525.

31. Catasus L, Gallardo A, Cuatrecasas M, Prat J. PIK3CA mutations in the kinase domain (exon 20) of uterine endometrial adenocarcinomas are associated with adverse prognostic parameters. Mod Pathol 2008, 21:131-139.

32. Janku F, Garrido-Laguna I, Petruzelka LB, Stewart DJ, Kurzrock R. Novel therapeutic targets in non-small cell lung cancer. J Thorac Oncol 2011, 6:1601-1612.

33. Nakayama $\mathbf{M}$, Hisatsune $J$, Yamasaki E, Isomoto $H$, Kurazono H, Hatakeyama M, Azuma T, et al. Helicobacter pylori VacA-induced inhibition of GSK3 through the PI3K/ Akt signaling pathway. J Biol Chem. 2009;284:1612-1619. 
34. Campbell IG, Russell SE, Choong DY, Montgomery KG, Ciavarella ML, Hooi CS, Cristiano BE, et al. Mutation of the PIK3CA gene in ovarian and breast cancer. Cancer Res. 2004;64:7678-81.

35. Yamamoto H, Shigematsu H, Nomura M, Lockwood WW, Sato M, Okumura N, Soh J, et al. PIK3CA mutations and copy number gains in human lung cancers. Cancer Res. 2008;68:6913-21.

36. Sobin L, Gospodarowicz M, Wittekind C, eds. TNM Classification of Malignant Tumours In: 7th ed. International Union Against Cancer (UICC). New York: Wiley, 2009.

37. Sakuramoto S, Sasako M, Yamaguchi T, Kinoshita T, Fujii M, Nashimoto A, Furukawa H, et al. ACTS-GC Group.
Adjuvant chemotherapy for gastric cancer with S-1, an oral fluoropyrimidine. N Engl J Med 2007;357:1810-1820.

38. Soh J, Okumura N, Lockwood WW, Yamamoto H, Shigematsu H, Zhang W, Chari R, et al. Oncogene mutations, copy number gains and mutant allele specific imbalance (MASI) frequently occur together in tumor cells. PLoS One 2009;4:e7464.

39. Lin CH, Huang MC, Li LH, Wu JY, Chen YT, Fann CS. Genome-wide copy number analysis using copy number inferring tool (CNIT) and DNA pooling. Hum Mutat. 2008;29:1055-62. 